\title{
Interactive comment on "The high frequency response correction of eddy covariance fluxes. Part 1: an experimental approach for analysing noisy measurements of small fluxes" by Toprak Aslan et al.
}

\author{
Marc Aubinet (Referee) \\ marc.aubinet@ulg.ac.be \\ Received and published: 15 January 2021
}

\section{General comments}

This paper is the first of two papers discussing different spectral corrections procedures for low pass filtering effects in eddy covariance systems. I read and reviewed both and found the present paper difficult to understand without reading the second (Peltola et al, also on AMT discussions). I thus recommend the authors to change the paper order and put the Peltola paper, which better stands alone, in first position and this one in 
second. I also made my reviews in this order and, for people who would be interested, I also recommend to read my review of the Peltola paper before this one.

This paper addresses two questions related to low pass frequency corrections for eddy covariance systems: one is the problem of power spectra contamination by white noise and the resulting difficulty to determine transfer function time constants when using the spectral approach (PSA); the second is the impact of transfer function shape and of time lag when using a cospectral approach (CSA).

Having never applied the PSA on low signal to noise ratios personnaly, I have no direct experience of the impact of noise on power spectra but I can imagine that this problem may be critical under low SNR. The method proposed by the authors seems to provide more accurate and less dispersed time constant estimates than the classical method proposed by Ibrom et al. 2007 (I suppose that Andreas supports this, as he is co-author of the paper). However, I didn't find the method very convincing as flux estimates obtained with this new approach did not appear much more accurate (Figure 8). In addition, I wonder about the feasibility of applying routinely this approach on "real world data" as the fit has to provide two parameters which could create convergence problems.

Above all, this makes me again wonder why one persists to follow the PSA while the CSA approach is not affected by noise.

I'm much more reluctant about the second comparison. The authors apply the CSA and compare three approaches, two based on non-synchronized cospectra and using either a Lorentzian curve or its square root and the third based on synchronized (time lag adjusted) cospectra using the square root of a Lorentzian. I was first puzzled by the use of non-synchronised cospectra, that appears a priori nonsense. In the present case, anyway, as the original (not attenuated) time series are not lagged, I suppose that the first approach (CSAH) could be thought as an application of the classical Lorentzian transfer function on a set on which the time lag introduced by signal attenuation would

Interactive comment

Printer-friendly version

Discussion paper 
have been ignored. The third one (CSA sqrt(H), sync) would then be those taking both attenuation and its derived time lag into account, following an approach described in the Peltola paper (Method 2). This comparison would then show that ignoring the time lag due to low pass filtering would lead to a time constant underestimation. If my interpretation is correct, I think that it should at least be explained by the authours.

Besides this, I don't see the interest of the second approach (CSA sqrt(H)) as it does not correspond to any used methodology. Contrary to what the authors suggest (P5L21), this comparison does not address the debate on transfer function shape: indeed the real question, very well synthesized by Peltola, was to determine, in the PSA, which transfer function should be applied on cospectra: the function itself or its square root (with the same time constant). This is not what was tested here as the Lorentzian and its square root were separately adjusted on the same data set resulting in a quite trivial result, i.e. the time constant of the CSA sqrt $(\mathrm{H})$ is about twice those of the CSA $\mathrm{H}$. An approach fitting a Lorentzian on synchronised time (CSA H, sync) would probably be more relevant as it would mimic the Method 1 of Peltola presently (and erroneously) recommended by the ICOS protocols. Anyway, I think that these comparisons are of limited interest as they overlap with results of Peltola.

Finally, I found both analyses (as well on PSA as on CSA) too much focused on time constants, which are not an objective per se when applying spectral correction procedures, and not enough on correction factors (not presented in the study) or fluxes. Correction factors are not presented and only cumulated fluxes are presented and (too) shortly discussed. I think that the real efficiency of the approaches can better be evaluated by looking half hourly fluxes and I suggest the authors to look at the regressions between half hourly fluxes obtained with the different approaches.

In the whole, the paper presents some promising results but some of the proposed comparisons are not relevant to my opinion and some analyses are insufficiently developed and not enough focused on the real poroduct of the spectral correction, i.e., the correction factors and the fluxes. 
Besides this, the paper is generally well written and presented but there are still some presentation problems that I point in the specific comments below. In conclusion, I AMTD think that the paper needs a major revision before publication.

\section{Specific comments}

P2L11 and P4L13: In the frequency space these operations are not convolutions but multiplications (a convolution in the time space corresponds to a multiplication in the frequency space and conversely).

P3L10: suppress the "be"

P3L11: Is this really critical? As the time lag is mainly determined by the set up, it could be determined on periods of larger flux and extrapolated. On the other hand, it's true that for small SNR, a time lag estimated by covariance maximisation, would systematically select the time lag associated to the highest flux and would not necessarily correspond to a "physical" maximum, which could lead to bias small fluxes.

P3L13: Yes but the paper showed that the impact on correction factors and fluxes was not critical if adequately accounted.

P5L21: See my general comments above. I think that the proposed experiment does not bring any relevant argument to this debate.

P5L29 and foll.: You also point below (P9L21) that, in the lbrom procedure, the boundaries for regression fitting are fixed by eye. This should be specified here as you consider this as a limit of the method.

P6L9: What does mean a "y axis intercept" in a log scale? In your case, this axis does even not appear in the figure!

P7L21-24: I had difficulties to understand this paragraph but the problem is maybe simply that you should refer to Eq (7) rather than Eq (4) on L24.

P7L32-P8L2: See general comments above. 
P9L17-23: This is somewhat a repetition of denoising description above (P5L29 and foll.) but only the 107 method is described. What about the A20 method? Clarify and AMTD avoid overlapping.

P12 Fig 4, P15 Fig 6: Spectra and cospectra were presented in function of the real frequency. Did you take the possible cospectral shift with wind velocity when taking ensemble averages?

P13Fig5: Red line and shaded area are confounded.

P14L10: the term "raw" is maybe not very well adapted here as it represents in fact the ideal cospectrum, without attenuation and noise.

P14L10: Was the cospectrum based on synchronized time series or not?

P14L10: It is in fact not so evident from the figure that noise contamination does not affect the cospectra shape. Do you refer to the fact that no linear increase is observed at high frequency? This could be specified.

\section{P14L12 and foll : See my general comment.}

P14L27: Yes but this is a problem that affects time constant determination but not correction factors and fluxes, as shown by Peltola.

P15L2: As I also pointed in my review of the Peltola paper, I'm not convinced by the use of relative errors on cumulated fluxes to evaluate the performance of correction methods. On one hand, relative errors are often not informative (the relative error on a zero flux would be infinite !), on the other hand computing errors on cumulated fluxes only would hinder error compensation (beteen night and day, for example). I prefer a comparison between half hourly fluxes (by taking the regression slope, for example).

P15L5: In view of Figure 8, it is not clear to me that PSA20 is more accurate than PSI07. I just see that it underestimates systematically the flux value but there is no clue that the bias is smaller. 
P15L6: Very close $(<1 \%)$ is only the case for the low time constants $(0.1$ and $0.2 \mathrm{~s})$. For higher time constants, it anyway reaches $2 \%$.

Interactive comment on Atmos. Meas. Tech. Discuss., doi:10.5194/amt-2020-478, 2020.

Interactive comment 